\title{
Terahertz detection in zero-bias InAs self-switching diodes at room temperature
}

\author{
Andreas Westlund, ${ }^{1}$ Paul Sangaré, ${ }^{2}$ Guillaume Ducournau, ${ }^{2}$ Per-Åke Nilsson, ${ }^{1}$ \\ Christophe Gaquière, ${ }^{2}$ Ludovic Desplanque, ${ }^{2}$ Xavier Wallart, ${ }^{2}$ and Jan Grahn ${ }^{1}$ \\ ${ }^{1}$ Department of Microtechnology and Nanoscience-MC2, Chalmers University of Technology, \\ SE-412 96 Gothenburg, Sweden \\ ${ }^{2}$ Institute of Electronics, Microelectronics and Nanotechnology UMR CNRS 8520, University of Lille, \\ CS 60069, 59652 Villeneuve d'Ascq, France
}

(Received 3 July 2013; accepted 6 September 2013; published online 23 September 2013)

\begin{abstract}
RF characterization of InAs self-switching diodes (SSDs) is reported. On-wafer measurements revealed no roll-off in responsivity in the range of $2-315 \mathrm{GHz}$. At $50 \mathrm{GHz}$, a responsivity of $17 \mathrm{~V} / \mathrm{W}$ and a noise-equivalent power (NEP) of $150 \mathrm{pW} / \mathrm{Hz}^{1 / 2}$ was observed for the SSD when driven by a $50 \Omega$ source. With a conjugately matched source, a responsivity of $34 \mathrm{~V} / \mathrm{W}$ and an NEP of $65 \mathrm{pW} / \mathrm{Hz}^{1 / 2}$ were estimated. An antenna-coupled SSD demonstrated a responsivity of $0.7 \mathrm{~V} / \mathrm{W}$ at $600 \mathrm{GHz}$. The results demonstrate the feasibility of zero-bias terahertz detection with high-electron mobility InAs SSDs up to and beyond $100 \mathrm{GHz}$. C 2013 AIP Publishing LLC.

[http://dx.doi.org/10.1063/1.4821949]
\end{abstract}

Development of zero-bias terahertz $(\mathrm{THz})$ detectors for imaging applications is driven by a search for higher bandwidth and sensitivity, the latter manifested through improved responsivity and noise performance. Compared to biased detectors, zero-bias detectors offer lower 1/f-noise and reduced dc power consumption. Room temperature zero-bias detection at frequencies above $100 \mathrm{GHz}$ is today realized with Schottky diodes ${ }^{1,2}$ or Sb-heterostructure backward diodes. ${ }^{3}$ An emerging device for zero-bias detection is the self-switching diode (SSD). ${ }^{4}$ The SSD is a field-effect device where a nanometer-sized constriction acts as a channel for the charge carriers. Similar to the Schottky diode or Sbheterostructure backward diode, the SSD detection principle is based on a current-voltage nonlinearity. First demonstrated in InGaAs, ${ }^{4}$ the SSD has since then been reported as a roomtemperature detector up to $1.5 \mathrm{THz}$ in GaAs. ${ }^{5} \mathrm{SSD}$ detection has also been published for indium-tin oxide, ${ }^{6} \mathrm{ZnO}^{7}$ and recently $\mathrm{GaN}^{8}{ }^{8}$ among other materials. Since InAs exhibits an electron mobility superior to all materials reported so far, higher operation frequency may be expected in an InAsbased SSD. Indeed, Monte Carlo (MC) simulations of InAs SSDs suggest a constant responsivity from DC to at least 2 THz. ${ }^{9}$ In this work, RF detection experiments for InAs SSDs are presented. On-wafer measurements up to $315 \mathrm{GHz}$ are presented, followed by detection at $600 \mathrm{GHz}$ in a free-space set-up with an antenna-coupled SSD. Responsivity and detector noise properties are reported.

SSDs are defined by a two-dimensional geometry in a semiconductor. In this work, the SSD was defined in an InAs/AlGaSb heterostructure containing a 2DEG. The lateral SSD design is shown in Figure 1. The channels, marked A in Figure 1, connect source to drain. The channels are separated by flanges, marked $\mathrm{B}$, which are connected to the drain contact. The SSD can be understood as a transistor where the flanges are gates, short-circuited to the drain. ${ }^{10}$ When a negative voltage is applied to the drain, the biased flanges reduce the carrier concentration in the channel thus increasing the (channel) resistance. A non-linear current-voltage relation arises, well-known for InAs SSDs. ${ }^{11}$ The modulation of the channel resistance is enhanced by reducing the channel width $W^{4}$ The effective channel width may be smaller than the physical channel width, due to depletion by surface charges. ${ }^{8}$

The epitaxial heterostructure used for InAs SSD fabrication was grown by molecular beam epitaxy (MBE). ${ }^{12}$ Similar structures are used for InAs/AlSb HEMT fabrication, ${ }^{13}$ which simplifies possible future MMIC integration. A $300 \mathrm{~nm} \mathrm{GaAs}$ buffer and a $1 \mu \mathrm{m}$ metamorphic AlSb buffer were grown on a semi-insulating GaAs substrate. The AlSb layer was covered by a $100 \mathrm{~nm} \mathrm{Al}_{80} \mathrm{Ga}_{20} \mathrm{Sb}$ buffer, a $15 \mathrm{~nm}$ InAs channel and a $13 \mathrm{~nm} \mathrm{Al}_{80} \mathrm{Ga}_{20} \mathrm{Sb}$ barrier, forming the non-intentionally doped quantum well. AlSb is known to be prone to oxidation. ${ }^{14}$ By choosing $\mathrm{Al}_{80} \mathrm{Ga}_{20} \mathrm{Sb}$ over AlSb in the top layers, chemical stability was enhanced. ${ }^{15}$ On top of the quantum well, a $4 \mathrm{~nm} \mathrm{In}_{52} \mathrm{Al}_{48} \mathrm{As}$ protection layer and a $5 \mathrm{~nm}$ Te-doped InAs contact layer were deposited. In fabrication of the SSD, the contact layer in the active part of the device was removed by wet etching, after which the protection

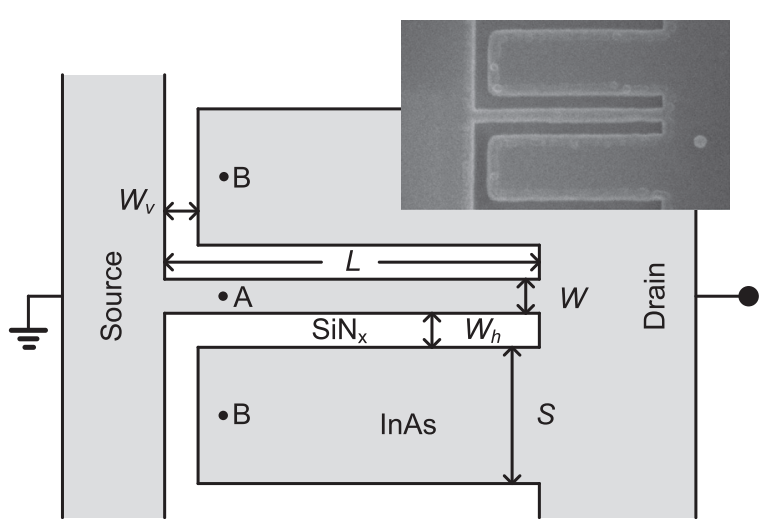

FIG. 1. Schematic view of the geometry of the InAs SSD. Trenches, pictured in white, were etched through the 2DEG of an InAs/Al80Ga20Sb quantum well. The inset shows a scanning electron microscopy of $100 \mathrm{~nm}$ wide etched trenches covered by $25 \mathrm{~nm}$ silicon nitride. 
layer prevented oxidation of the $\mathrm{Al}_{80} \mathrm{Ga}_{20} \mathrm{Sb}$ barrier. Trenches were patterned with e-beam lithography and etched with a $\mathrm{Cl}$ :Ar reactive ion etch (RIE), reaching through the InAs channel and forming the geometry of the 2DEG depicted in Figure 1. ${ }^{12}$ Without breaking the vacuum, $25 \mathrm{~nm}$ silicon nitride was grown in a plasma-enhanced chemical vapor deposition process to protect the $\mathrm{Al}_{80} \mathrm{Ga}_{20} \mathrm{Sb}$ barrier against oxidation and provide electrical passivation. The inset of Figure 1 shows the etched trench at this stage. Later, the etched trenches were completely filled with silicon nitride for enhanced encapsulation and increased field coupling between the flanges and channels. Room-temperature Hall measurements of the passivated heterostructure with removed cap layer yielded an electron concentration of $1.5 \times 10^{12} \mathrm{~cm}^{-2}$ and an electron mobility of $26000 \mathrm{~cm}^{2} / \mathrm{Vs}$, which are representative numbers for similar heterostructures. ${ }^{16}$ All SSDs were designed with channel length $L=1100 \mathrm{~nm}$, separation $S=350 \mathrm{~nm}$, vertical trench width $W_{v}=100 \mathrm{~nm}$, and horizontal trench width $W_{h}=100 \mathrm{~nm}$. Channel width $W$ and number of channels $N$ were varied.

The non-linearity of the I-V characteristics is the basis for RF detection in SSDs. The asymmetric current defined as $I(V)+I(-V)$ is plotted in Figure 2 for a device with $W=35 \mathrm{~nm}$ and $N=43$. The inset shows the I-V characteristics, which appears to be linear. While the asymmetric current is of the same magnitude as for other non-linear detectors such as Sb-heterostructure backward diodes, ${ }^{17}$ it constitutes only around $3 \%$ of the total current in the InAs SSD. However, it is this small asymmetry that makes the InAs SSD a detector.

An RF detection experiment was carried out on-wafer using a vector network analyzer as the signal source. To reduce the resistance of the device and improve the match to $50 \Omega$, designs with $N=43$ were used. $W$ was varied from 35 to $120 \mathrm{~nm}$. Three different setups were used for the frequency bands 2-50 GHz, 140-220 GHz, and 240-315 GHz, all following the same principle: The power from the source was measured with a calorimetric power meter for each frequency setting. By subtracting the loss of the RF probes, the available power at the probe tip was found. The power incident to the device was $1.7-8.5 \mu \mathrm{W}$, delivered by a $50 \Omega$ source. Resulting DC detection voltage was measured through a bias-tee with a high-resistance load. The

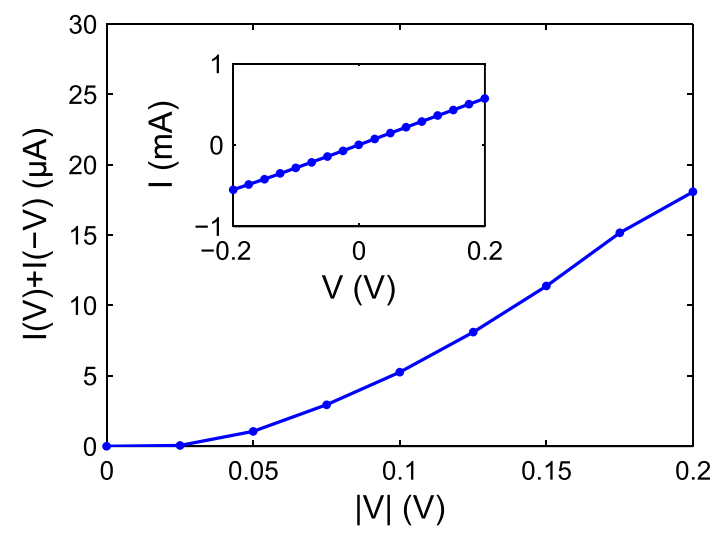

FIG. 2. The asymmetric component $I(V)+I(-V)$ of the I-V characteristics of an InAs SSD with $W=35 \mathrm{~nm}$ and $N=43$. The insert shows the I-V characteristics of the InAs SSD.

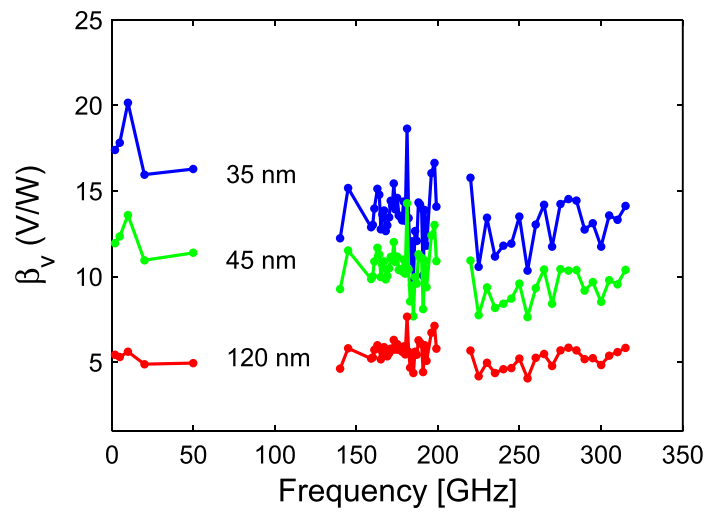

FIG. 3. Measured $\beta_{v}$ versus frequency for three different $W$.

unmatched responsivity $\beta_{v}$ was found as the ratio of incident power and measured voltage.

Figure 3 shows $\beta_{v}$ versus frequency for three different $W$. No significant roll-off was observed in the measured frequency bands. A $\beta_{v}>10 \mathrm{~V} / \mathrm{W}$ at $2-315 \mathrm{GHz}$ was achieved for $W=35 \mathrm{~nm}$. For larger $W, \beta_{v}$ was lower, but showed similar frequency dependence. A more detailed study of the $W$ dependence of $\beta_{v}$ is shown in Figure 4, performed at $50 \mathrm{GHz}$ with an incident power of $3.2 \mu \mathrm{W}$. The highest $\beta_{v}=17 \mathrm{~V} / \mathrm{W}$ was achieved for $W=35 \mathrm{~nm}$, the smallest $W$ investigated.

Using a matching network between the source and the detector would increase the responsivity. Figure 4 shows the responsivity with a conjugately matched source, $\beta_{\text {opt }}$, versus $W$. Using the measured $S_{11}$ and $\beta_{v}, \beta_{o p t}$ was found as $\beta_{\text {opt }}=\beta_{v} /\left(1-\left|S_{11}\right|^{2}\right)$. Comparing $\beta_{\text {opt }}$ to $\beta_{v}$, it is seen that for $W=120 \mathrm{~nm}, \quad \beta_{\text {opt }} \approx \beta_{v}$, whereas for $W=35 \mathrm{~nm}$, $\beta_{\text {opt }} \approx 2 \beta_{v}$. This is due to the increased diode impedance for small $W$ and thus an increased mismatch to $50 \Omega$. Moreover, Figure 4 suggests that both $\beta_{v}$ and $\beta_{\text {opt }}$ would improve if $W$ was decreased further.

Also shown in Figure 4 is $\beta_{o p t}$ as predicted from I-V characterizations. Predictions matched the measured $\beta_{\text {opt }}$ closely, both in absolute values and in $W$-dependence. The prediction of $\beta_{\text {opt }}$ was done by fitting a fifth-order polynomial to the measured I-V characteristics for $V \in[-0.2,0.2] \mathrm{V}$. Then $\beta_{\mathrm{opt}}$ was found as

$$
\beta_{\text {opt }}=\frac{1}{2} R_{0} \gamma
$$

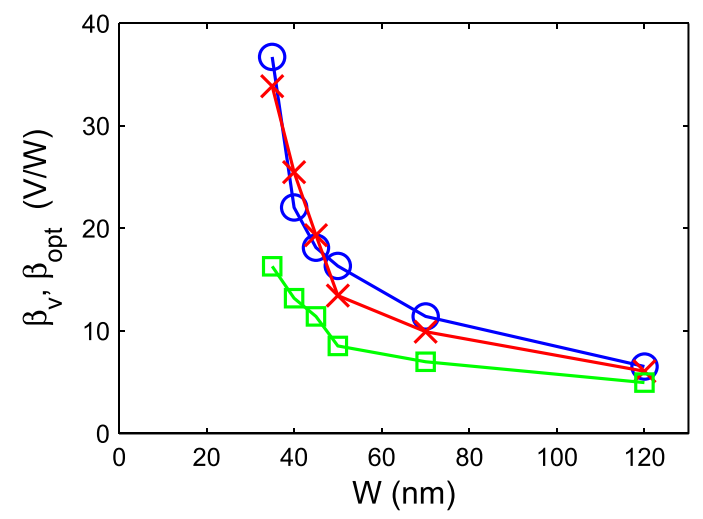

FIG. 4. Measured $\beta_{v}$ (squares) and $\beta_{\text {opt }}$ (crosses) at $50 \mathrm{GHz}$ versus $W$, and predictions of $\beta_{\text {opt }}$ based on I-V analysis (circles). 


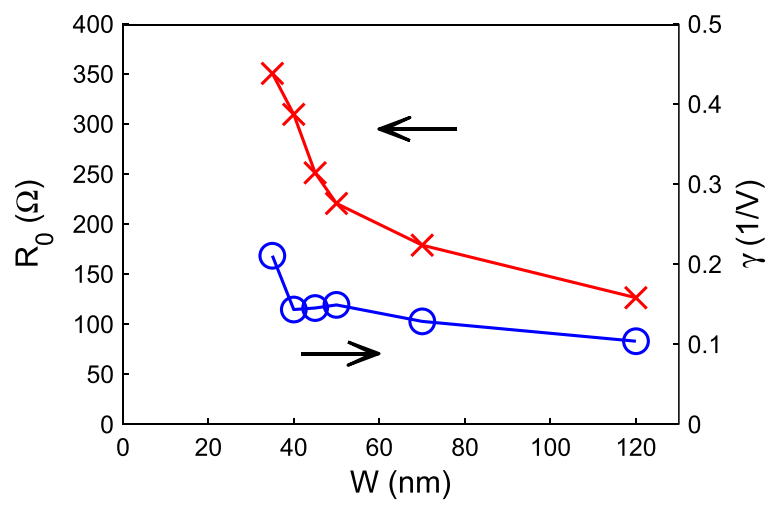

FIG. 5. $W$-dependence of $R_{0}$ (crosses) and $\gamma$ (circles) as found by I-V measurements.

where $R_{0}=1 /\left.(d I / d V)\right|_{V=0}$ is the zero-bias resistance and $\gamma=\left(d^{2} I / d V^{2}\right) /\left.(d I / d V)\right|_{V=0}$ is the curvature. ${ }^{18}$ From $W=120$ to $35 \mathrm{~nm}, \beta_{\text {opt }}$ increased from 6.1 to $34 \mathrm{~V} / \mathrm{W}$, corresponding to a factor 5.6 increase. By studying $R_{0}$ and $\gamma$, as shown in Figure 5, it is seen that the improvement in $\beta_{\text {opt }}$ was due to an increase in both $R_{0}$ and $\gamma$. The increase in $R_{0}$ was a factor 2.8 (from 126 to $350 \Omega$ ) and in $\gamma$ a factor 2.1 (from 0.10 to $0.21 \mathrm{~V}^{-1}$ ). The increase in $\gamma$ is important since it shows that the increase in $\beta_{\text {opt }}$ is partly due to an increased modulation of the current through each channel, and not merely to an increased $R_{0}$. An increased $R_{0}$ increases $\beta_{o p t}$, but makes matching increasingly difficult and has a less straight-forward effect on the detectors noise properties.

For zero-bias detectors, the dominating noise process at low incident power is Johnson-Nyquist noise, which has been shown for SSDs specifically. ${ }^{19}$ Hence, the rms voltage spectral density across the terminals is $V_{n}=\sqrt{4 K T R}_{0}$, where $k$ is Boltzmann's constant and $T$ the physical temperature. A central figure of merit for a detector is the noise equivalent power (NEP), representing the input power for which the output equals the noise floor. The NEP of the SSD when driven by a conjugately matched source was defined as $\mathrm{NEP}_{\text {opt }}$ and found as

$$
N E P_{\text {opt }}=\sqrt{4 K T R_{0}} / \beta_{\text {opt }},
$$

where $T=295 \mathrm{~K}$. Similarly, the NEP for a diode driven by a $50 \Omega$ source was found as $N E P_{v}=\sqrt{4 K T R_{0}} / \beta_{v} . \mathrm{NEP}_{v}$ and $\mathrm{NEP}_{\text {opt }}$ versus $W$ is plotted in Figure 6, where $T=295 \mathrm{~K}$. Both $\mathrm{NEP}_{v}$ and $\mathrm{NEP}_{o p t}$ decreased when $W$ was lowered,

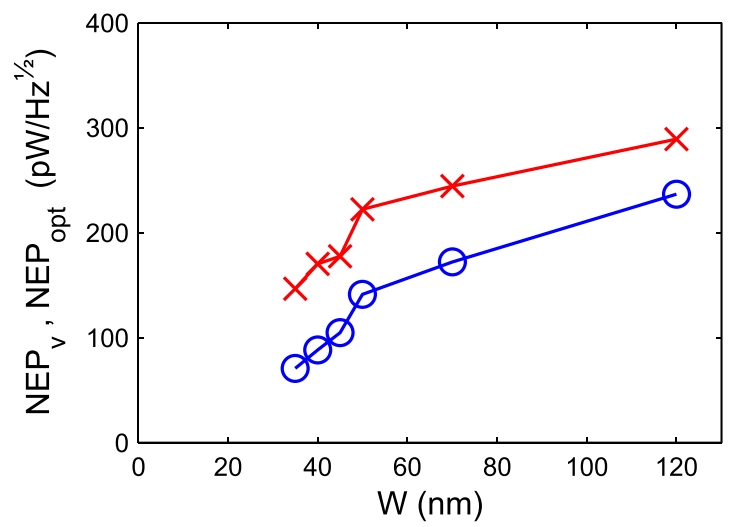

FIG. 6. Measured $\mathrm{NEP}_{v}$ (crosses) and $\mathrm{NEP}_{\text {opt }}$ (circles) at $50 \mathrm{GHz}$ versus $W$.

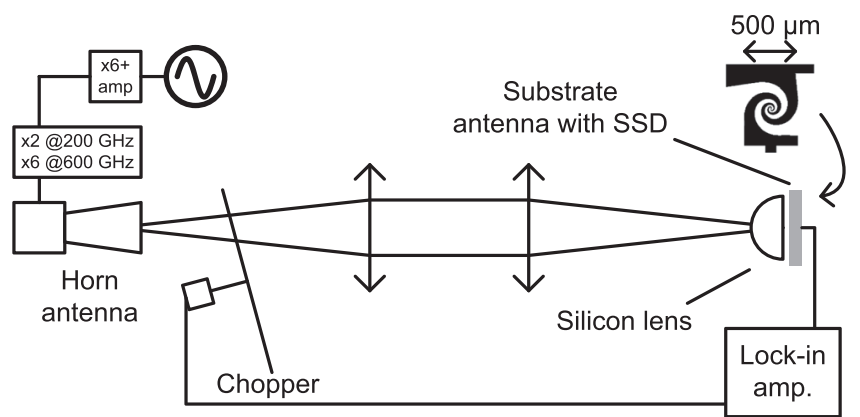

FIG. 7. Setup for free-space measurements of an antenna-coupled InAs SSD at 200 and $600 \mathrm{GHz}$.

despite the increase in $R_{0}$. The lowest values were achieved for $W=35 \mathrm{~nm}$, for which $\mathrm{NEP}_{v}=150 \mathrm{pW} / \mathrm{Hz}^{1 / 2}$ and $\mathrm{NEP}_{\text {opt }}=65 \mathrm{pW} / \mathrm{Hz}^{1 / 2}$. It can be seen from Figure 6 that by further reducing $W$ a reduction of both $\mathrm{NEP}_{v}$ and $\mathrm{NEP}_{\text {opt }}$ can be expected.

To investigate if InAs SSDs can function as detectors at higher frequencies, an antenna-coupled SSD was used with the free-space setup shown in Figure 7. A detector system was built with an InAs SSD fabricated with a circularly polarized spiral substrate antenna, ${ }^{20}$ for which the simulated impedance was $90 \Omega$. The SSD was designed with $N=11$ and $W=65 \mathrm{~nm}$. From I-V characterization, $\beta_{\text {opt }}=53 \mathrm{~V} / \mathrm{W}$ was expected. A silicon lens was placed firmly against the backside of the substrate with the SSDs. The output voltage was measured with a lock-in amplifier, thus presenting a high DC-load. On the transmitter side, the vertically polarized beam was radiated through a conical horn antenna. By replacing the receiver with an Erickson PM4 power meter with a horn antenna, the incident average power was measured to $1.15 \mathrm{~mW}$ and $7 \mu \mathrm{W}$, at $200 \mathrm{GHz}$ and $600 \mathrm{GHz}$, respectively. The responsivities of the detector were $2.1 \mathrm{~V} / \mathrm{W}$ and $0.70 \mathrm{~V} / \mathrm{W}$, respectively. Compensating for polarization mismatch $(50 \%)$ and antenna mismatch $(\approx 50 \%), \beta_{\text {opt }}$ was calculated to $8.4 \mathrm{~V} / \mathrm{W}$ and $2.8 \mathrm{~V} / \mathrm{W}$ at $200 \mathrm{GHz}$ and $600 \mathrm{GHz}$, respectively. This shows that InAs SSDs can function as detectors at at least $600 \mathrm{GHz}$.

InAs self-switching diodes were fabricated and characterized as detectors in the range $2-315 \mathrm{GHz}$, in which no roll-off of responsivity was observed. At $50 \mathrm{GHz}$, the highest responsivity and lowest NEP observed were $17 \mathrm{~V} / \mathrm{W}$ and 150 $\mathrm{pW} / \mathrm{Hz}^{1 / 2}$, measured with a $50 \Omega$ source and no matching network. With a conjugately matched source, $34 \mathrm{~V} / \mathrm{W}$ and 65 $\mathrm{pW} / \mathrm{Hz}^{1 / 2}$ are expected. Finally, detection was confirmed at $600 \mathrm{GHz}$ using an antenna-coupled SSD in a free-space setup. These results demonstrate the feasibility of InAs SSDs in zero-bias detection up to and beyond $100 \mathrm{GHz}$.

This work has been sponsored by the European Commission FP7 through the ROOTHz project ICT-2009243845.

\footnotetext{
${ }^{1}$ L. Liu, J. L. Hesler, H. Xu, A. W. Lichtenberger, and R. M. Weikle, IEEE Microw. Wirel. Compon. Lett. 20, 504 (2010).

${ }^{2}$ D. Schoenherr, C. Bleasdale, T. Goebel, C. Sydlo, H. L. Hartnagel, R. Lewis, and P. Meissner, in 35th International Conference on Infrared, Millimeter and Terahertz Waves, Rome, Italy, 5-10 September 2010 (IEEE, Piscataway, NJ, USA, 2010).
} 
${ }^{3}$ N. Su, Z. Zhang, J. N. Schulman, and P. Fay, IEEE Electron Device Lett. 28, 336 (2007).

${ }^{4}$ A. M. Song, M. Missous, P. Omling, A. R. Peaker, L. Samuelson, and W. Seifert, Appl. Phys. Lett. 83, 1881 (2003).

${ }^{5}$ C. Balocco, S. R. Kasjoo, X. F. Lu, L. Q. Zhang, Y. Alimi, S. Winnerl, and A. M. Song, Appl. Phys. Lett. 98, 223501 (2011).

${ }^{6}$ J. Kettle, R. M. Perks, and R. T. Hoyle, Electr. Lett. 45, 79 (2009).

${ }^{7}$ M. Y. Irshaid, C. Balocco, Y. Luo, P. Bao, C. Brox-Nilsen, and A. M. Song, Appl. Phys. Lett. 99, 092101 (2011).

${ }^{8}$ P. Sangaré, G. Ducournau, B. Grimbert, V. Brandli, M. Faucher, C. Gaquière, A. Íñiguez-de-la-Torre, I. Íñiguez-de-la-Torre, J. F. Millithaler, J. Mateos, and T. González, J. Appl. Phys. 113, 034305 (2013).

${ }^{9}$ I. Iñiguez-de-la-Torre, H. Rodilla, J. Mateos, D. Pardo, A. M. Song, and T. González, J. Phys.: Conf. Ser. 193, 012082 (2009).

${ }^{10}$ M. Åberg, J. Saijets, A. Song, and M. Prunnila, Phys. Scr. T114, 123 (2004).

${ }^{11}$ A. Westlund, G. Moschetti, H. Zhao, P.-A. Nilsson, and J. Grahn, in 24th International Conference on Indium Phosphide and Related Materials, Santa Barbara, CA, USA, 27-30 August 2012 (IEEE, Piscataway, NJ, USA, 2012), pp. 65-68.

${ }^{12}$ A. Westlund, G. Moschetti, P. - $\AA$. Nilsson, J. Grahn, L. Desplanque, and $\mathrm{X}$. Wallart, in 25th International Conference on Indium Phosphide and
Related Materials, Kobe, Japan, 19-23 May 2013 (IEEE, Piscataway, NJ, USA, 2013).

${ }^{13}$ G. Moschetti, N. Wadefalk, P. - $\AA$. Nilsson, M. Abbasi, L. Desplanque, X. Wallart, and J. Grahn, IEEE Microw. Wirel. Compon. Lett. 22, 144 (2012).

${ }^{14}$ A. Olivier, T. Gehin, L. Desplanque, X. Wallart, Y. Roelens, G. Dambrine, A. Cappy, S. Bollaert, E. Lefebvre, M. Malmkvist, and J. Grahn, in 20th International Conference on Indium Phosphide and Related Materials, 25-29 May 2008, Versailles, France (IEEE, Piscataway, NJ, USA, 2008).

${ }^{15}$ G. Moschetti, E. Lefebvre, M. Fagerlind, P. -Å. Nilsson, L. Desplanque, X. Wallart, and J. Grahn, Solid-State Electron. 87, 85 (2013).

${ }^{16}$ G. Tuttle, H. Kroemer, and J. H. English, J. Appl. Phys. 65, 5239 (1989).

${ }^{17}$ N. Su, R. Rajavel, P. Deelman, J. N. Schulman, and P. Fay, IEEE Electron Device Lett. 29, 536 (2008).

${ }^{18}$ A. M. Cowley and H. O. Sorensen, IEEE Trans. Microwave Theory Tech. 14, 588 (1966).

${ }^{19}$ C. Balocco, S. R. Kasjoo, L. Q. Zhang, Y. Alimi, and A. M. Song, Appl. Phys. Lett. 99, 113511 (2011).

${ }^{20}$ A. D. Semenov, H. Richter, H.-W. Hubers, B. Gunther, A. Smirnov, K. S. Il'in, M. Siegel, and J. P. Karamarkovic, IEEE Trans. Microwave Theory Tech. 55, 239 (2007). 